\title{
Penser l'éducation chorale en la pratiquant. Approche métathéâtrale du Philoctète de Sophocle
}

Sophie Klimis

\section{(2) OpenEdition}

1 Journals

Édition électronique

URL : https://journals.openedition.org/edl/3153

DOI : $10.4000 /$ edl. 3153

ISSN : 2296-5084

Éditeur

Université de Lausanne

\section{Édition imprimée}

Date de publication : 24 septembre 2020

Pagination : $59-78$

ISBN : 978-2-940331-74-1

ISSN : 0014-2026

\section{Référence électronique}

Sophie Klimis, « Penser l'éducation chorale en la pratiquant. Approche métathéâtrale du Philoctète de Sophocle ", Études de lettres [En ligne], 313 | 2020, mis en ligne le 24 septembre 2021, consulté le 25 septembre 2021. URL : http://journals.openedition.org/edl/3153; DOI : https://doi.org/10.4000/edl. 3153 


\section{PENSER L'ÉDUCATION CHORALE EN LA PRATIQUANT. APPROCHE MÉTATHÉÂTRALE DU PHILOCTĖTE DE SOPHOCLE}

En mobilisant les témoignages d'Aristophane et de Platon sur le rôle de la tragédie dans la paideia des jeunes gens, et en considérant comme "symptômes" la virulence de leurs critiques, il s'agira de proposer l'hypothèse d'une stylisation de l'initiation éphébique dans le Philoctète, qui met en évidence l'altération/aliénation de l'identité, le devenir-autre comme phase transitoire, mais considérée comme nécessaire dans le processus du devenir citoyen ${ }^{1}$.

Entre "initiation" rituelle des choreutes et "éducation" politique des spectateurs, faut-il choisir? ${ }^{2}$

Dans un article fameux, John Winkler avait émis l'hypothèse selon laquelle le chœur tragique aurait été composé d'éphèbes, jeunes gens âgés de 18 à 20 ans effectuant un service militaire avant d'être définitivement intégrés dans le corps civique ${ }^{3}$. Chantant et dansant, déguisés en femmes, en esclaves, en vieillards ou encore en étrangers, les éphèbes auraient joué à faire les anti-citoyens pour devenir des citoyens, dans le cadre d'une performance tragique devant désormais être comprise

I. Une première version de ce texte a été présentée lors du colloque «Pourquoi la métathéâtralité? » organisé en 2007 à l'Université Diderot-Paris 7 par Florence Dupont.

2. S. Klimis, «Le lyrique dans la tragédie grecque».

3. J. Winkler, "The Ephebes' Song». En évoquant le fait que certaines parmi les meilleures places étaient réservées aux éphèbes, Winkler considère que ce devaient être les meilleurs d'entre eux qui étaient choisis pour former les chœurs. 
comme un rite d'initiation. Si l'intérêt de cette hypothèse a été reconnu par certains commentateurs ${ }^{4}$, ils en ont restreint la portée en estimant qu'elle ne pouvait pas être prouvée 5 . Il n'existe en effet aucun texte faisant explicitement référence à un chœur d'éphèbes ${ }^{6}$. Qui plus est, l'institution de l'éphébie militaire n'est elle-même pas attestée au $\mathrm{V}^{\mathrm{e}}$ siècle, les sources principales - le fragment sur La Constitution des Athéniens d'Aristote et la stèle d'Acharnes sur laquelle est gravé le serment des éphèbes - datant du IV ${ }^{\mathrm{e}}$ siècle?

Seule Florence Dupont s'est risquée à prolonger cette hypothèse. Selon elle, le "devenir autre" des éphèbes aurait "une valeur purement fonctionnelle ${ }^{8}$ : le travestissement des choreutes serait le marqueur rituel du passage de l'enfance à l'âge adulte, "une étape dans la formation de l'adulte athénien " ${ }^{9}$. Le sens ultime de cette expérience de l'altérité aurait été d'être "une épreuve psychologique de maîtrise de soi. Comme ils apprennent au banquet à maîtriser l'ivresse, les jeunes gens du chœur apprennent à maîtriser la musique de l'aulos ${ }^{10}$. Ainsi, la fonction rituelle du chœur d'éphèbes barrerait la route à toute interprétation politique de la tragédie: «bien loin d'être un espace de débat [...] les

4. P. Wilson la qualifie d'"unproven [...] but the material Winkler assembles in the process of making this imaginative case [...] represents an absolutely vital contribution" (The Athenian Institution of the Khoregia, p. 17). S. Goldhill considère pour sa part que le rôle central accordé au défilé des éphèbes orphelins de guerre dans le cadre des cérémonies précédant les concours tragiques, ainsi que le nombre de personnages de fiction qui sont des éphèbes (Oreste, Hippolyte, etc.) attestent d'un lien privilégié entre cette classe d'âge et les performances tragiques ("The Great Dionysia and Civic Ideology», p. 124 sq.). Il considère cependant qu'il est délicat de supposer qu'il en allait déjà de même au V $\mathrm{V}^{\mathrm{e}}$ siècle, comme le fait pourtant $\mathrm{A}$. Pickard-Cambridge, The Dramatic Festivals of Athens, p. 60 sq.

5. P. Vidal-Naquet, «Retour au chasseur noir», p. 247 sq.

6. Winkler se base surtout sur l'étude d'un cratère datant de la fin du $\mathrm{V}^{\mathrm{e}}$ siècle, qui représente acteurs et membres du chœur leurs masques à la main : tous les masques sont barbus, mais tous les porteurs de masques de satyres sont glabres, à la différence des acteurs.

7. Cf. Ch. Pelekidis, Histoire de l'éphébie attique; P. Siewert, "The Ephebic Oath in Fifth-Century Athens». Tous deux, ainsi que P. Vidal-Naquet ( Retour au chasseur noir», p. 118 sq.) et Goldhill, ("The Great Dionysia and Civic Ideology», p. 124) estiment cependant qu'une pratique de l'éphébie non institutionnalisée et le serment des éphèbes devaient déjà exister au $\mathrm{V}^{\mathrm{e}}$ siècle.

8. F. Dupont, Aristote ou le vampire du théâtre occidental, p. 297.

9. Ibid.

Io. Ibid., p. 298. 
tragédies ne témoigneraient d'aucune réflexion critique sur la société grecque ou sur la guerre, sur les femmes, la servitude, la politique ${ }^{11}$.

Ce constat anti-politique est aussi anti-poétique: il permet à Dupont de parachever sa déconstruction du primat de la "raison narrative" imposé par le prisme de lecture aristotélicien. Rappelons que Dupont interprète $L a$ Poétique d'Aristote comme une vaste opération idéologique visant à détruire la performance tragique en la transformant en texte. Dès lors, l'hypothèse du chœur d'éphèbes lui permet de considérer que les muthoi tragiques «ne sont que le prolongement de la fonction rituelle ${ }^{12}$ : d'après elle, les intrigues tissées par l'enchaînement des épisodes seraient de simples «simulations pédagogiques à l'usage des éphèbes, qui se concluent toujours par des maximes d'une sagesse basique ${ }^{13}$. L'interprétation se veut polémique, ce qui fait tout autant sa force que sa limite. Elle vise à juste titre à revaloriser l'importance de la musique et du rituel, en mettant en question l'«évidence» du primat du texte, voire sa quasi-sacralisation. Mais, en déniant à la tragédie toute fonction politique, critique et réflexive, elle soutient une position intenable. Car ce qu'il s'agit précisément de comprendre, c'est cette articulation si singulière du rituel et du politique qui caractérisait la tragédie à Athènes.

Rappelons que les tragédies étaient "performées» dans le cadre d'un concours intégré dans la fête religieuse des Grandes Dionysies. Le théâtre n'est donc pas né de la sécularisation du rite dionysiaque, il en faisait partie. Ces festivités en l'honneur de Dionysos étaient aussi éminemment politiques, car elles avaient pour particularité d'impliquer de nombreux citoyens: il revenait à un magistrat, l'archonte-roi, de choisir les tragédies qui allaient concourir et de veiller à la bonne organisation de toute la manifestation. À chaque poète était associé un chorège choisi parmi les citoyens aisés, qui était chargé de financer l'entretien du chœur. Le jury était composé de dix citoyens. Si les acteurs étaient des professionnels, les chœurs semblent avoir été composés de citoyens qui étaient exemptés de leurs charges politiques et militaires pendant toute la durée des répétitions et jusqu'à l'unique performance, soit durant un

II. Ibid., p. 297, 299.

I2. Ibid., p. 299.

I3. Ibid. 
an ${ }^{14}$. Chanter et danser dans un chœur, à Athènes, c'était donc faire de la politique, au même titre que délibérer au Conseil ou voter à l'Assemblée, tout autant que c'était participer à un rituel pour Dionysos.

Cet étrange mélange n'a rien à voir avec ce que nous entendons aujourd'hui par l'expression de "théologico-politique». Le "religieux» ne servait pas à "fonder» le politique en lui donnant une assise idéologique, la tragédie ayant pour fonction de mettre en crise les fondements de la démocratie (l'autonomie, l'isonomie, etc.) en les questionnant de manière radicale. Ce "questionnement» de l'institution démocratique avait pour particularité de faire appel aussi bien à la raison qu'aux émotions et de s'adresser aussi bien aux spectateurs qu'à ceux qui performaient la tragédie. didaskalos

Ainsi, la tragédie fut bien plus que le "miroir», fût-il «brisé», auquel la cité se serait "réfléchie» elle-même ${ }^{15}$. Car elle ne se réduisit pas à la représentation d'une "fiction". Sa portée critique tint plutôt au fait d'être, en acte, le seul "fondement" possible pour une démocratie directe: celui qui institue, chaque année, la possibilité de sa propre destitution ${ }^{16}$. C'est pourquoi tant les interprétations "politiques» de la tragé$\operatorname{die}^{17}$, que celles qui sont exclusivement "rituelles», manquent l'essentiel, qui réside dans le complexe entre-tissage de ces deux dimensions. Nicole Loraux, à tort associée au camp des «ritualistes», l'avait bien vu:

Nous éprouvons la plus grande difficulté à penser une configuration conflictuelle qui soit productrice d'une forme forte, parfaitement une, insécable en quelque sorte. Aussi, pour fuir le conflit, choisissons-nous un pôle plutôt que l'autre: pour avoir voulu montrer que toute lecture purement politique de la tragédie était réductrice, je crains d'avoir donné l'impression de substituer une lecture antipolitique ${ }^{18}$.

Dès lors, l'hypothèse du chœur d'éphèbes est intéressante, en ce qu'elle fournit une piste pour comprendre cette articulation spécifique du politique et du rituel. Elle permet de saisir l'importance du rôle joué par la construction philosophique d'un objet "théorique» qui a contribué à

I4. E. Csapo, W. J. Slater, The Context of Ancient Drama, p. 349-368.

I5. P. Vidal-Naquet, Le miroir brisé.

I6. S. Klimis, "L'harmonie discordante».

17. Ch. Meier, De la tragédie comme art politique.

18. N. Loraux, La voix endeuillée, p. 120 sq. 
effacer l'importance de cette articulation: une "tragédie" réduite à un divertissement purement fictionnel et étrangement coupée de ses dimensions politiques et rituelles, tant dans La République de Platon que dans La Poétique d'Aristote.

Le poète tragique, le poète comique ou le philosophe: qui est le didaskalos des citoyens athéniens? ${ }^{19}$

On peut problématiser dans une autre direction que Dupont le lien entre le chœur d'éphèbes et la théorisation aristotélicienne de la tragédie. En effet, c'est à Aristote que Winkler emprunte deux arguments pour fonder sa thèse: les noms qui spécifient les fonctions des membres du chœur tragique sont des termes militaires utilisés pour désigner les membres d'une phalange d'hoplites. Ainsi, le coryphée est aussi appelé hègemôn (le «commandant») et les choreutes qui sont directement à ses côtés sont des parastates - un terme désignant le soldat placé dans un rang à côté d'un autre ${ }^{20}$. Aristote mentionne aussi que «la seconde année de l'éphébie, une assemblée est tenue au théâtre et les éphèbes y font une démonstration au peuple de ce qui concerne leur mise en rang et ils reçoivent de la cité un bouclier et une lance ${ }^{21}$. La description est assez floue que pour y voir la danse chorale, forme stylisée et fictionnalisée de la marche d'une phalange, qui en conserve la formation rectangulaire ${ }^{22}$. Pourtant, Aristote n'en dit mot dans La Poétique, où il diminue l'importance du chœur, qui doit selon lui se comporter comme un personnage et participer au déroulement de l'intrigue, se soumettant au primat de la logique narrative ${ }^{23}$.

$\mathrm{Du}$ «lieu» théorique politique à celui de la poétique, la description philosophique de la tragédie a donc métamorphosé cette dernière, en

19. S. Klimis, «Le drame de la narration».

20. Aristote, Politique, III, 4, 1277a.

21. Aristote, La Constitution des Athéniens, XLII, 4.

22. J. Winkler, "The Ephebes' Song», p. 57. D. Wiles critique Winkler en défendant la forme circulaire du chœur tragique (Tragedy in Athens, p. 93 sq). Csapo et Slater (The Context of Ancient Drama) apportent des témoignages décisifs en faveur de la formation rectangulaire du chœur au $\mathrm{V}^{\mathrm{e}}$ siècle, liée à la forme de l'orchestra.

23. S. Klimis, "Le lyrique dans la tragédie grecque»; F. Dupont, Aristote ou le vampire du théatre occidental, p. 51. 
effaçant toute trace de son insertion dans la vie politique et religieuse de la cité, pour la réduire à n'être plus qu'une «œuvre». Il faut prendre la mesure de ce que signifie la focale exclusivement «technique» de $\mathrm{La}$ Poétique: le poète n'est plus en rien un éducateur, il est désormais un artisan. La tragédie, telle que reformatée par Aristote, n'est plus un acte rituel d'initiation ni un acte politique de mise en question de l'institution héritée. Elle est un «divertissement» qui doit susciter «le plaisir qui lui est propre», basé sur la terreur et la pitié ${ }^{24}$. Ainsi, l'entreprise théorique de La Poétique parachève la critique platonicienne de la tragédie envisagée comme poésie mimétique dans La République de Platon ${ }^{25}$. En effet, Simon Goldhill touche juste lorsqu'il pose que:

Plato's hostility to the poets is to a large degree due to the status of authority held by poets as teachers or controllers of knowledge - the role which Plato wishes to appropriate for philosophy alone ${ }^{26}$.

C'est le statut de didaskalos reconnu par tous aux poètes que le philosophe entend annexer, en s'auto-instituant seul maître de vérité et de vertu. Or, si aux dires du Socrate de La République, Homère est le didaskalos de la Grèce toute entière (X, 606e), le poète tragique est celui de la cité démocratique athénienne.

Le poète comique Aristophane, lui-même rival à ce titre du poète tragique, nous en fournit un témoignage ironique: dans Les Grenouilles, Eschyle considère que ses pièces enseignent aux citoyens à être nobles et vaillants (1013-1017) et s'oppose à Euripide qui prétend leur avoir appris à "réfléchir, voir, comprendre, aimer se retourner, machiner, à soupçonner le mal et à considérer toutes choses" (957-958). Tous deux s'accordent cependant sur le fait que les poètes tragiques «rendent les hommes meilleurs dans les cités» (1009-1010). Si l'on a toujours identifié le public des spectateurs comme étant le destinataire de cet enseignement tragique, un autre passage laisse entendre qu'il pourrait bien aussi viser les choreutes, si l'on tient compte de l'hypothèse éphébique:

24. Aristote, Poétique 14, 1453b10-14. Cf. S. Klimis, Le statut du mythe dans la Poétique d'Aristote.

25. S. Klimis, Archéologie du sujet tragique, p. 40-43.

26. S. Goldhill, "The Great Dionysia and Civic Ideology", p. 108. Cf. aussi A. Wilson-Nightingale, Genres in Dialogue, p. 60-67; S. Klimis, Archéologie, p. 25-40. 
[...] le poète est tenu de cacher le vice, non de le mettre au jour, de le produire sur la scène, de l'enseigner. Car pour les petits enfants, l'éducateur c'est le maître d'école, pour les jeunes gens (hèbôsi), c'est le poète. Nous avons l'absolu devoir de ne dire que des choses honnêtes (1053-1055).

Aristophane fait explicitement des poètes tragiques les éducateurs des «jeunes gens». Qui plus est, en créant cet agôn imaginaire au sujet de l'objet de l'éducation tragique, il présente cette dernière comme une «évidence» du sens commun pour ses spectateurs. Enfin, si Dionysos descend aux Enfers pour en ramener le meilleur poète tragique, c'est pour l'aider à lutter contre un mal «nouveau» qui se serait abattu sur la cité d'Athènes et qui n'est autre que... l'éducation prodiguée par Socrate (1482-1499). Cette pièce nous dévoile par ailleurs que le poète comique entendait lui-même entrer en lice, tentant in fine de fonder la supériorité de l'éducation qu'il délivre aux citoyens. Il semble donc qu'Aristophane nous ait transmis le témoignage d'une situation de crise: celle d'un agôn qui eut lieu à Athènes, afin de ravir aux poètes tragiques leur titre d'éducateurs des citoyens.

Dès lors, il me semble qu'il faut réentendre la critique platonicienne de la mimèsis au livre III de La République dans le sillage de l'hypothèse éphébique, renforcée par le témoignage d'Aristophane. À ma connaissance, il n'y a pas d'interprétation qui propose de voir dans l'examen du recours à la mimèsis dans l'éducation des gardiens de la cité «imaginaire» («en paroles», dit Socrate), une manière de critiquer indirectement l'éducation chorale des citoyens athéniens. Or, le danger de la «contamination" mimétique de l'âme pointé par Socrate semble bien s'appliquer aux éphèbes-choreutes. Rappelons en effet que les éphèbes athéniens, qualifiés de peripoloi, assumaient une charge de garde de la cité dans des fortins situés à ses frontières. Dès lors, les "gardiens» de la cité imaginée par Socrate et ses compagnons pourraient, par cette fonction, désigner les éphèbes d'une manière aussi indirecte qu'explicite pour les lecteurs contemporains de Platon.

Venons-en à la critique. Selon Socrate, la mimèsis, si elle a commencé dès l'enfance, se sédimente en èthos: elle devient une seconde nature, qui transforme le corps, la voix et la pensée de celui qui la pratique (III, 395e1 et 395d3). La mimèsis est en effet définie comme le fait de "simuler» être quelqu'un d'autre. Celui qui la pratique est donc celui qui se 
dissimule en "énonçant quelque discours comme s'il était quelqu'un d'autre» (III, 393c). La poésie mimétique produirait donc des hommes doubles, voire multiples, donc contradictoires, irrecevables dans la cité imaginaire "où chacun ne fait qu'une seule chose" (III, 397d-e). Le constat de Socrate, selon lequel la cité imaginaire est la meilleure possible, "surtout compte tenu de l'exclusion de la poésie mimétique" (X, 595a), montrerait ainsi en quoi la cité construite dans le discours philosophique vise à discréditer le rôle éducatif des poètes. En effet, il est frappant de constater que tous les objets de la mimèsis énumérés par Socrate correspondent aux rôles des chœurs tragiques et comiques (femmes, esclaves, animaux, etc.). Or, selon Socrate, les plaintes et les cris des chants tragiques et comiques enseignés par les poètes feraient courir aux choreutes le risque de véritablement se transformer en femmes, en esclaves et en animaux. L'emphase est ironique. Ce dont il s'agit, c'est plutôt que l'âme "s'effémine», qu'elle "s'animalise» en passant sous le contrôle de sa partie irrationnelle que Socrate décrit comme étant avide de larmes. Le khorodidaskalos est ainsi présenté comme celui dont «l'enseignement» fortifie la partie irrationnelle de l'âme et détruit le logos tant des choreutes que des spectateurs. Le processus est bien distinct dans les deux cas, mais le résultat est le même. Le seul "antidote" est une mimèsis d'un nouveau genre: celle qui n'imite que des hommes nobles et vertueux... qui ressemblent étrangement à Socrate. On peut ainsi considérer que c'est le dialogue platonicien lui-même qui se désigne indirectement comme étant ce pharmakon du théâtre, en tant qu'il est destiné à être lu et non pas joué. Dès lors, la lecture assumerait le rôle central de "garde» dans la conduite de l'âme par la partie rationnelle. L'engagement du corps, dans la danse et le chant choral, est au contraire vu comme ce qui joue un rôle central dans le «relâchement» de l'âme au profit de l'irrationnel. On comprend donc que la critique des poètes dépasse largement leur éviction du cadre de la cité «imaginaire» de $L a$ République. Car c'est l'acte de fondation de la «différence» philosophique qui se joue dans la victoire à l'agôn des éducateurs.

\section{Lecture métathéâtrale du Philoctète de Sophocle}

Je n'ai ici qu'effleuré les conséquences qui découleraient d'une validation de l'hypothèse éphébique pour ce qui concerne l'interprétation de la 
critique adressée par les philosophes aux poètes. Mon but est d'explorer cette hypothèse en montrant qu'on peut trouver dans les textes tragiques des éléments permettant de la fonder, à condition de les envisager sous un angle métathéâtral. Je précise tout d'abord que j'entends le métathéâtre au sens où Mark Ringer l'a défini :

Metatheater or metadrama means drama within drama as well as drama about drama [...] Metatheater, however, extends far beyond the boundaries of the literal «play-within-the-play». It encompasses all forms of theatrical self-referentiality. These may include role playing, various forms of self-conscious reference to dramatic convention and other plays, and the many ways in which a playwright may toy with the perceived boundaries of his or her craft. Other elements of metatheatrical phenomena include ritual or ceremonial enactments within the play and the rupturing of dramatic illusion ${ }^{27}$.

Je voudrais donc m'attacher à montrer que la «mutation ${ }^{28}$ du personnage de Néoptolème dans le Philoctète de Sophocle reflète la transformation de soi qui résulte de l'initiation éphébique envisagée tant dans ses aspects militaires que dans la participation à un chœur tragique.

Seule tragédie conservée jusqu'à nous sans personnage féminin, le Philoctète est aussi l'une des rares qui mette en scène un éphèbe. Le jeune Néoptolème, fils du défunt Achille, est un éphèbe orphelin de guerre, sur le point de se joindre à l'expédition de Troie et donc d'intégrer activement l'armée: ce statut le met d'emblée en relation avec le défilé des orphelins de guerre, moment clé du contexte civique dans lequel s'inséraient les tragédies ${ }^{29}$. Alors que la plupart des tragédies supposent une forme d'aliénation des choreutes par leur travestissement en "Autre" du citoyen (femme, esclave, etc.), le Philoctète présente l'intéressante particularité de faire jouer à un homme adulte un personnage d'éphèbe dont le devenir fictionnel représente ce que seraient réellement en train

27. M. Ringer, Electra and the Empty Urn, p. 7 sq. Je suis toutefois d'accord avec N. W. Slater, Spectator Politics, p. 3, qui problématise la notion de «rupture d'illusion".

28. P. Vidal-Naquet, «Le Philoctète de Sophocle et l'éphébie», p. 155 souligne qu'il s'agit d'un exemple unique dans l'œuvre de Sophocle de mutation du héros tragique.

29. S. Goldhill, «The Great Dionysia and Civic Ideology», p. 119. 
de vivre en même temps les choreutes, figurant quant à eux des personnages fictionnels de marins adultes ${ }^{30}$.

Rappelons l'intrigue: un oracle a prédit que Troie ne pourrait être prise qu'avec l'arc de Philoctète, abandonné sur une île déserte par Ulysse. Or, c'est le même Ulysse, détesté de Philoctète, qui est chargé de ramener l'arc, avec l'aide de Néoptolème, à qui il demande de convaincre Philoctète de s'embarquer avec lui en usant d'une ruse. Il doit faire croire à Philoctète qu'il a lui aussi subi un préjudice de la part des Achéens, en se présentant donc comme leur ennemi. Comme Achille, le père de Néoptolème, fut par ailleurs l'ami de Philoctète, Ulysse pense que Philoctète aura toutes les raisons de faire confiance à Néoptolème et de tomber dans son piège en acceptant de partir avec lui. Se soumettant à la ruse ordonnée par Ulysse, les marins jouent à composer un personnage à l'intérieur de la pièce (role-playing-within-the-role) et leur duplicité va jusquau parjure et au sacrilège ${ }^{31}$, ce qui constitue un cas unique dans le corpus tragique. En effet, pour accréditer le pouvoir persuasif du mensonge de Néoptolème lorsqu'il se dit ennemi des Atrides, le chœur en appelle à la déesse Terre "mère de Zeus lui-même", comme témoin des souffrances de son maitre (391-402). Comme le souligne Ringer, "the chorus is, in effect, singing and dancing a rather sacrilegious lie» ${ }^{32}$. Le chœur chante aussi un péan sacrilège à Hypnos (827-832), qui a pour fonction de tromper Philoctète ${ }^{33}$.

L'approche métathéâtrale permet d'accorder un statut à ces chants impies. Ringer voit en Ulysse un playwright-within-the-play ${ }^{34}$ : auteur et metteur en scène de sa ruse, qui assigne à chaque personnage un rôle, allant même jusqu'à régler l'entrée en scène d'un marin transformé en marchand fictif. Tout ceci est machiné pour le seul Philoctète, spectateur malgré lui d'une fiction qu'il prendra pour la réalité. On peut aller plus loin. Le prologue peut en effet être interprété comme la mise en fiction d'un dialogue entre un khorodidaskalos et un éphèbe récalcitrant à devenir choreute:

30. La pièce fut jouée un an après le rétablissement de la démocratie par les marins qui renversèrent l'oligarchie des Quatre Cents.

3I. M. Ringer, Electra and the Empty Urn, p. 111.

32. Ibid.

33. S. Esposito, The Chorus in Sophocles, p. 84 sq.

34. M. Ringer, Electra and the Empty Urn, p. 107. 
U.: il faut employer la ruse. Ainsi tu lui déroberas ses armes invincibles. Je sais bien, enfant, que par nature, tu n'es pas fait pour tenir un langage de ce genre, ni pour inventer un artifice. Pourtant, car c'est vraiment douce chose que de tenir entre ses mains le prix de la victoire, aie cette audace; nous nous montrerons justes une autre fois. Aujourd'hui, sans scrupules, livre-toi à moi pour un court instant de la journée, et ensuite, pendant tout le reste du temps, sois proclamé le plus pieux de tous les mortels.

N.: ce que je souffre à entendre, je déteste aussi l'exécuter; je ne suis pas né pour agir par ruse perfide [...] je suis prêt à enlever cet homme par la violence, non par la ruse (77-88).

Lorsqu'Ulysse pose que la ruse est le seul moyen pour dérober les armes de Philoctète, il enjoint à Néoptolème de jouer un rôle dans sa "pièce»: simuler d'être la victime des Atrides et l'ennemi d'Ulysse. Mais la référence à «l'artifice» mobilisé à un "moment de la journée», passage obligé pour tenir en main le prix de la victoire, semble aussi pouvoir être directement mise en relation avec le contexte du concours tragique. La honte de Néoptolème reflèterait ainsi celle du choreute, forcé de se travestir le temps de la performance, alors que son andreia - masculinité culturelle $\mathrm{du}$ citoyen et de ses valeurs - le pousse à s'illustrer dans l'exploit guerrier et la vie politique. De façon générale, on peut supposer qu'une forme de violence était ainsi imposée à l'èthos du choreute, contraint de se costumer en femme, en esclave, en étranger ou ici en marin - membre de la classe censitaire la plus pauvre - et d'apparaître devant des milliers de spectateurs. Etre un anticitoyen le temps de la performance tragique passait aussi pour le choreute par l'obligation d'accomplir des actes inciviques, comme pleurer et se lamenter - une loi de Solon l'interdisait aux hommes - ou des actes impies, comme ici, chanter l'hymne et le péan fallacieux.

Cette violence imposée à l'èthos du citoyen peut s'expliquer si on la situe dans le contexte rituel de l'initiation des éphèbes, comme l'allusion au serment qui précède directement le passage cité le laisse supposer. Ulysse justifie que Néoptolème soit le seul à pouvoir jouer le rôle imposé par sa ruse par le fait qu'il n’a pas "prêté le serment" (72). VidalNaquet avait déjà signalé que ce serment des alliés achéens peut être interprété comme une allusion au serment éphébique que Néoptolème n'a pas encore prêté et qui justifie qu'il se soumette à la ruse d'Ulysse, ce qui lui permettra d'accomplir son premier exploit guerrier en capturant 
Philoctète ${ }^{35}$. Mais cette référence à l'éphébie militaire me semble aussi pouvoir être élargie au contexte tragique et indiquer que le comportement impie et incivique du chœur ne serait justifiable que dans le cadre d'un rite d'initiation.

C'est donc le personnage de Néoptolème, en tant que fictionnalisation de l'éphèbe-choreute, qui justifie métathéâtralement les paroles impies réellement prononcées par les éphèbes choreutes déguisés en marins. Être impies le temps de la performance serait une étape nécessaire pour ensuite pouvoir être appelés «les plus pieux des mortels tout le reste du temps». La traduction littérale du vers 82 «nous nous dévoilerons justes en retournant sur nos pas", pourrait renforcer cette hypothèse en faisant référence à l'exodos, la sortie du chœur, une fois initié à son nouveau statut de citoyen/hoplite. On aurait donc là une explicitation métathéâtrale du fait que la participation des meilleurs éphèbes au chœur tragique aurait constitué une sorte de rite collectif avant que tous prêtent le serment éphébique qui les fait accéder au statut de citoyen à part entière. Le masque des marins, défenseurs de la démocratie dans l'actualité récente, redoublerait l'impiété rituelle d'une signification politique.

L'armée achéenne représentée dans cette pièce ne figure ni la cité athénienne ni son opposé. Comme Goldhill l’a signalé, les tragédies ne se limitent pas à une simple inversion des normes de la cité athénienne. Elles construisent des fictions qui en questionnent les fondements mêmes ${ }^{36}$. L'armée achéenne me semble constituer une simulation fictionnelle du même type, qui donne à voir ce que serait une cité sans concitoyenneté sociale, c'est-à-dire sans philia. Cette armée est en effet régie par des rapports de hiérarchie stricts et elle n'a qu'une seule règle: l'obéissance absolue de l'inférieur à son supérieur. Ainsi, les premières paroles des marins sont pour demander à leur chef ce qu' ils doivent faire (135-143). Néoptolème est quant à lui soumis aux ordres d'Ulysse, qui obéit lui-même aux Atrides. Dans cette structure pyramidale de domination, on est loin du partage du pouvoir qui caractérise la démocratie athénienne.

Dès les premiers vers, cette armée se singularise par un trait marquant: l'abandon de l'un des siens sur une île déserte (1-5). Pour un public athénien, l'armée achéenne figure donc la fiction d'une armée

35. P. Vidal-Naquet, "Le Philoctète de Sophocle et l'éphébie», p. 164.

36. S. Goldhill, "The Great Dionysia and Civic Ideology», p. 127 sq. 
d'hommes adultes qui auraient trahi l'élément le plus important du serment des éphèbes: «je n'abandonnerai pas mon voisin là où je serai en rang». L'existence de ce serment à l'époque où fut joué le Philoctète semble attestée par le fait que cette pièce problématise l'articulation des cinq injonctions qui le constituent: ne pas déshonorer les armes sacrées, ne pas abandonner son camarade de rang, défendre la religion, obéir aux dirigeants et aux lois, honorer les cultes ancestraux des pères. Par exemple, si Philoctète a été abandonné, c'est afin de défendre la religion, car ses plaintes incessantes empêchaient de sacrifier aux dieux; et si Néoptolème désobéit à Ulysse, c'est pour ne pas abandonner son compagnon et afin d'honorer les dieux puisque Philoctète s'est fait son suppliant.

Dans ce contexte, il est permis de supposer que le personnage de Philoctète, incarnation de l'homme sauvage ${ }^{37}$, compose la fiction monstrueuse d'un homme adulte figé dans le stade transitoire de l'éphébie. Philoctète, sur le point de quitter l'île avec Néoptolème, lui demande de l'accompagner une dernière fois dans sa caverne: «je te conduirai, mon mal exige ton assistance (se xumparastatèn)»(674-675). Littéralement, Philoctète demande à Néoptolème d'être son parastate, son compagnon d'armes, le soldat placé à côté de lui dans la phalange. Cette réplique peut aussi s'entendre dans le contexte de la performance tragique: Philoctète se présenterait alors comme hègemôn, chef de chœur s'adressant à Néoptolème comme à son parastate, choreute situé directement à ses côtés. S'ensuit le premier stasimon, où le chœur chante sa pitié pour Philoctète en l'absence de ce dernier, ce qui laisse supposer qu'il est sincère. Dans la seconde strophe, cette pitié se concentre sur la description de la solitude de Philoctète, qui résonne métathéâtralement comme la description d'un homme exclu de l'assistance procurée par l'insertion dans une phalange:

Il était à lui-même son propre voisin, il n'avait pas de marche réglée, de pas cadencé, il n’avait près de lui personne pour l'assister dans sesmaux, personne auprès de qui il aurait pu résister au gémissement que lui arrachait sa blessure» (691-694).

37. P. Vidal-Naquet, «Le Philoctète de Sophocle et l'éphébie», p. 157-161 ; Ch. Segal, Tragedy and Civilization, p. 292-327. 
Cette plainte se poursuit par la description de l'état de sauvagerie de Philoctète, qui est aussi celle de la vie quotidienne du chasseur noir, l'éphèbe péripolos:

Il rampait ici et là, il se traînait en décrivant des circuits, comme un enfant tenu à l'écart, sans nourrices aimantes, là où sa dextérité lui permettait de trouver un chemin, lorsque cessait le mal qui le rongeait (700-705).

La seconde strophe poursuit cette description de Philoctète en éphèbe péripolos et dévoile que cet état de sauvagerie ne peut être que transitoire et initiatique, car il est déshumanisant:

Sans prendre pour se nourrir la semence de la terre sacrée, ni rien de ce que nous mangeons nous autres, hommes industrieux, sauf quand parfois avec les traits ailés de son arc, il procurait quelque aliment à son estomac. Pendant un temps de dix années, il n’a pas connu la joie des coupes de vin! Il ne buvait que de l'eau stagnante s'il en découvrait quelque part (708-717).

Rappelons en effet que, dès Homère, les «mangeurs de pains» désignent les «êtres humains». Dans la seconde antistrophe, la rencontre avec Néoptolème est présentée comme la délivrance de Philoctète, car le retour de ce dernier dans sa patrie est assimilé à sa réintégration dans l'humanité. Cette réhumanisation est placée sous le patronage d'Héraclès "porteur du bouclier de bronze» (726). Selon Vidal-Naquet, c'est l'Héraclès hoplite et non plus chasseur qui est désigné par cette épithète $^{38}$. J'en déduis que l'humanité se définit par l'insertion dans une phalange, c'est-à-dire par la participation à la vie politique en tant que citoyen. Tant qu'il n'a pas reçu son bouclier d'hoplite, l'éphèbe n'est pas encore tout à fait humain. Je propose donc de considérer que dans ce stasimon, les choreutes se lamentent rituellement sur leur propre sort et chantent l'horreur que leur inspirerait leur statut d'éphèbes, au cas où il ne serait pas dépassé par leur intégration dans la phalange et donc dans la cité des êtres humains. Ce stasimon dévoile ainsi que dans l'imaginaire collectif citoyen, l'éphèbe constitue une menace pour l'ordre de la cité, si sa "sauvagerie» n'est pas domptée et canalisée. Platon s'en fera à sa

38. P. Vidal-Naquet, «Le Philoctète de Sophocle et l'éphébie», p. 169. 
manière l'écho dans La République, en considérant que discipliner le thumos des gardiens est la finalité essentielle de leur éducation.

Le second épisode, où Néoptolème reste présent aux côtés de Philoctète durant tout l'accès de son mal, est la transposition fictionnelle de l'injonction éphébique à ne pas abandonner son camarade de rang. Mobilisant un lexique militaire, Philoctète implore Néoptolème de «ne pas le laisser seul» (809), ce qui est à entendre au sens de rester avec lui dans le rang de la phalange (810); et Néoptolème répète qu'il tiendra sa place $(809,813)$. Si l'exploit du Néoptolème éphèbe était censé être la capture de l'homme sauvage, celui de Néoptolème hoplite sera de tenir ferme aux côtés de son camarade dans son combat contre la maladie. Soulignons que Philoctète dit à Néoptolème ne pas vouloir le contraindre en le liant par un serment (811), et que le jeune homme lui répond que c'est Thémis, déesse de la justice, qui ne lui permet pas de s'en aller (812). Néoptolème, l'éphèbe-hoplite en devenir, et Philoctète, le mâle-éphèbe sauvage, composent ainsi la fiction d'une phalange à deux. Cette dernière dévoile que le serment des éphèbes ne tire pas sa force contraignante de sa dimension contractuelle, ni même de la crainte des dieux, mais de la philia pour l'homoios, de l'amitié pour le semblable qui n'est pas l'identique, et pourtant comme un autre soi-même.

Philoctète et Néoptolème composent par ailleurs aussi la fiction d'un kommos. Dans cet épisode, les deux protagonistes, qui s'étaient précédemment qualifiés de parastate et de hègemôn, font alterner en échos les cris de douleur de Philoctète et les lamentations de Néoptolème. Le pappapappapai (754) de Philoctète est un cri de douleur qui résonne aussi comme une interpellation directe de Néoptolème, puisqu'on y entend le pai («enfant») dont Philoctète s'était précédemment servi pour désigner Néoptolème (750). Le Iô, Iô, dustène su ("Oh, que tu es infortuné!») (v. 759) de Néoptolème lui répond par l'intériorisation de cette douleur, jusqu'à aboutir à la fusion des deux personnages au vers 806 où Néoptolème dit "souffrir depuis longtemps des maux de Philoctète". Ceci est d'autant plus étonnant que ce kommos subvertit les règles du genre. Comme il n'y a aucun mort à pleurer, il ne peut y avoir de chant de deuil. Philoctète se lamente tout seul sur son sort présent, et reste sourd aux paroles du chœur, qui le rend responsable de ses propres malheurs et tente de le raisonner en lui enjoignant de le suivre à Troie. Le chœur reste étonnamment distancié durant tout le kommos et ne semble plus éprouver aucune pitié pour Philoctète. Son manque d'empathie 
est tel que dans sa dernière réplique, le coryphée dit qu'il serait reparti depuis longtemps s'il ne craignait Néoptolème (1218-1221). Alors que la fonction traditionnelle du kommos est de happer le protagoniste dans le chœur, le chœur le maintient ici à distance. Métathéâtralement, le chœur d'éphèbes trahit son futur serment en formulant le vœu d'abandonner l'un des siens, tandis que Philoctète tient ferme son rang en solitaire (1197), ce qui est un oxymore.

La rupture de Philoctète avec l'armée, entamée par cette séparation d'avec le chœur, culmine dans le projet de retour dans la patrie que Néoptolème a finalement accepté. Caractérisant toujours leur relation par une terminologie militaire (1396-1409), les deux hommes deviennent donc une phalange de déserteurs qui tiendra ferme face à l'armée achéenne, désormais ennemie. Contrairement à une lecture répandue, le «retournement» de Néoptolème ne constitue pas un retour aux valeurs épiques paternelles. Néoptolème trahit aussi la mémoire de son père en désertant: dans un cadre épique, il aurait nécessairement dû prendre Troie pour perpétuer la gloire de son père. Cette situation intenable est résolue par l'apparition d'Héraclès en deus ex machina. En commençant par faire le récit de ses propres souffrances, le héros divinisé lie la gloire au pathos et pas seulement à la praxis. La gloire de Philoctète sera elle aussi liée à ses souffrances présentes et à leur dépassement dans l'exploit guerrier accompli en commun avec Néoptolème. À ce dernier, Héraclès prédit: «ni toi sans lui tu n'auras la force de t'emparer de la plaine de Troie, ni lui sans toi. Mais comme deux lions partageant le même sort, celui-ci devra te garder et toi tu devras le garder» (1433-1437). La prédiction du dieu est une forme stylisée du serment des éphèbes, qui ne fait qu'entériner le nouvel ordre de valeurs dessiné par l'interrelation de Philoctète et de Néoptolème. Aux rapports hiérarchiques de subordination dans l'armée et à la recherche de gloire individuelle du héros épique, se substitue une identité civique basée sur l'interaction, la réciprocité, l'entraide, la complémentarité et la solidarité. La parole du dieu fait ainsi voir en quoi la philia de Néoptolème et Philoctète est d'une intensité telle, qu'elle dépasse le cadre des valeurs épiques. Cette "amitié» rendue visible par la fiction est l'affect citoyen au fondement du projet démocratique: c'est elle qui permet à tous les citoyens de se considérer comme des homoioi, avant même que de se définir par rapport à leur appartenance clanique ou topologique. 


\section{Conclusion}

Le personnage de Néoptolème me semble constituer la mise en fiction de la mutation identitaire attendue d'un éphèbe au cours de son initiation à l'andreia du citoyen hoplite adulte. D'abord soumis à la ruse imposée par la communauté (chasse ou danse travestie forcée), l'éphèbe expérimente une violence rituelle nécessaire: celle de la honte de soi qui découle de comportements imposés d'anti-hoplites, impies et inciviques, comme chasseur noir ou comme choreute travesti. Transitoirement aliéné, l'éphèbe choreute semble donc bien subir une «épreuve psychologique de maitrise de soi ", mais la formation rectangulaire en phalange garantit le maintien de l'ordre civique. C'est donc le rythme du corps marchant et dansant qui constitue le cadrage nécessaire à ce que la déliaison de l'identité puisse avoir lieu sans basculer dans l'effondrement, alors que ce dernier menace la parole, sans cesse modulée du cri au discours. Cette honte de soi est nécessaire pour permettre l'ouverture à la pitié pour autrui, par la prise de conscience d'un vécu commun. Pitié terrifiante et extraordinaire (oiktos deinos, 965) qui a rendu Néoptolème capable de déserter l'armée et de trahir la mémoire de son père, mais qui doit nécessairement être ressentie pour que s'élabore la philia au fondement de la cité démocratique. Cette tragédie dévoile donc la dimension d'affect nécessaire à la création de la solidarité des membres d'une phalange, d'un chœur et d'une cité, car ces différentes formes de koinônia n'existent que dans et par l'interaction incessante de leurs membres. Comme le résume Thucydide, «la cité, c'est les hommes» ${ }^{39}$.

Ainsi, l'hypothèse éphébique permet de complexifier la compréhension de la paideia tragique, par-delà les dichotomies simplificatrices des Modernes: elle est initiation à Dionysos et éducation critique à la citoyenneté, tant pour les choreutes et les acteurs que pour les spectateurs, conjoignant la découverte de "soi-même comme un autre» et l'apprentissage de la phronèsis à travers la mobilisation d'une gamme d'affects fondamentaux.

Sophie KLimis

Université Saint-Louis-Bruxelles

39. Thucydide, La Guerre du Péloponnèse (VII, LXXVII, 7). 


\section{BIBLIOGRAPHIE}

\section{Textes}

Aristophane, Les Grenouilles, texte établi par Victor Coulon, traduit par Hilaire Van Daele, Paris, Les Belles Lettres, 1942.

Aristote, La Poétique, texte, traduction, notes par Roselyne Dupont-

Roc et Jan Lallot, Paris, Seuil, 1980.

—, La Constitution des Athéniens, texte établi et traduit par Georges Mathieu et Bernard Hassoulier, revu par Claude Mossé, introduction et notes de Claude Mossé, Paris, Les Belles Lettres, 2002. —, Politique, texte établi et traduit par Jean Aubonnet, Paris, les Belles Lettres, 1991 (1960).

Platon, La République, texte établi et traduit par Émile Chambry, Paris, Les Belles Lettres, 1946.

Sophocle, Philoctète, texte établi et traduit par Paul Masqueray, Paris, Les Belles Lettres, 1942.

Thucydide, La Guerre du Péloponnèse, texte établi et traduit par Louis Bodin et Jacqueline de Romilly, Paris, Les Belles Lettres, 2003 (1955).

\section{Travaux}

Calder William, "Sophoclean Apologia: Philoctetes", Greek and Roman Byzantines Studies, 12 (1971), p. 153-174.

Csapo, Éric, Slater, William J., The Context of Ancient Drama, Ann Arbor, The University of Michigan Press, 1994.

Dupont, Florence, Aristote ou le vampire du théâtre occidental, Paris, Aubier, 2007.

Esposito, Stephen J., The Chorus in Sophocles: The Tension of Foreground and Background in "Philoctetes" and "Tranchiniae", Michigan, 1990. 
Goldhill Simon, "The Great Dionysia and Civic Ideology", in John J. Winkler, Froma Zeitlin (eds), Nothing to do with Dionysos? Athenian Drama in its Social Context, Princeton, Princeton University Press, 1990, p. 97-129.

Klimis, Sophie, Le statut du mythe dans la Poétique d'Aristote. Les fondements philosophiques de la tragédie, Bruxelles, Ousia, 1997.

—, Archéologie du sujet tragique, Paris, Kimé, 2003.

-, «Le lyrique dans la tragédie grecque. Chants d'une pensée aporétique", in Antonio Rodriguez, André Wyss (éds), Le chant et l'écrit lyrique: poétiques et performances, Berne, Peter Lang, 2009, p. 197-215.

—, "Le drame de la narration: une invention philosophique?» in Arielle Meyer MacLeod, Michèle Pralong (éds), Raconter des histoires. Quelle narration au théâtre aujourd'hui?, Genève, Métis Press, 2012, p. 57-84.

—, "L'harmonie discordante: un schème pour penser radicalement l'institution de la démocratie", Raisons politiques, 75 (2019/3), p. 95-109.

Loraux, Nicole, La voix endeuillée. Essai sur la tragédie grecque, Paris, Gallimard, 1999.

Meier, Christian, De la tragédie comme art politique, Paris, Les Belles Lettres, 1991.

Pickard-Cambridge, Arthur Wallace, The Dramatic Festivals of Athens, Oxford, Clarendon Press, 1988 (1953).

Pelenidis, Chrysis, Histoire de l'éphébie attique des origines à 31 av. J.-C., Paris, Éditions de Boccard, 1962.

Ringer, Mark, Electra and the Empty Urn. Metatheater and Role Playing in Sophocles, Chapel-Hill/London, University of North Carolina Press, 1998.

Segal, Charles, «Philoctetes: The Myth and the Gods», in Id., Tragedy and Civilization. An Interpretation of Sophocles, Oklahoma, The University of Oklahoma Press,1981, p. 328-361.

Siewert, Peter, "The Ephebic Oath in Fifth-Century Athens", Journal of Hellenic Studies, 97 (1977), p. 102-111.

Slater, Niall W., Spectator Politics. Metatheatre and Performance in Aristophanes, Philadelphia, University of Pennsylvania Press, 2002. 
Vidal-Naquet, Pierre, «Le chasseur noir et l'origine de l'éphébie athénienne», in, Jean-Pierre Vernant, Pierre Vidal-Naquet, La Grèce ancienne 3. Rites de passage et transgressions, Paris, 1992 (1981), p. 119-147.

—, Le miroir brisé: tragédie athénienne et politique, Paris, Les Belles Lettres, 2001 (1989).

—, "Le Philoctète de Sophocle et l'éphébie», in Jean-Pierre Vernant, Pierre Vidal-Naquet, La Grèce ancienne 3. Rites de passage et transgressions, Paris, 1992 (1972), p. 149-177.

—, "Retour au chasseur noir", in Jean-Pierre Vernant, Pierre VidalNaquet, La Grèce ancienne 3. Rites de passage et transgressions, Paris, 1992 (1989), p. 215-251.

Wiles, David, Tragedy in Athens. Performance Space and Theatrical Meaning, Cambridge, Cambridge University Press, 1997.

Wilson, Peter, The Athenian Institution of the Khoregia. The Chorus, the City and the Stage, Cambridge, Cambridge University Press, 2000.

Wilson-Nightingale, Andrea, Genres in Dialogue. Plato and the Construct of Philosophy, Cambridge, Cambridge University Press, 2000 (1995).

Winkler, John J., "The Ephebes'Song: Tragoidia and Polis», in John J. Winkler, Froma Zeitlin (eds), Nothing to do with Dionysos? Athenian Drama in its Social Context, Princeton, Princeton University Press, 1990, p. 20-62. 